\title{
Structure and intermolecular vibrations of 7-azaindole-water 2:1 complex in a supersonic jet expansion: Laser-induced fluorescence spectroscopy and quantum chemistry calculation ${ }^{\#}$
}

\author{
MONTU K HAZRA ${ }^{\mathrm{a}, \mathrm{b}}$, MOITRAYEE MUKHERJEE ${ }^{\mathrm{c}}, \mathrm{V}^{\mathrm{R} A M A N A T H A N}{ }^{\mathrm{a}, \mathrm{d}}$ and \\ TAPAS CHAKRABORTY $Y^{\mathrm{c}, *}$ \\ a Department of Chemistry, Indian Institute of Technology Kanpur, Kanpur 208 016, India \\ bepartment of Chemistry and Biochemistry, University of California, San Diego, La Jolla, San Diego, \\ CA 93093-0314, USA \\ ${ }^{\mathrm{c}}$ Department of Physical Chemistry, Indian Association for the Cultivation of Science, \\ Jadavpur, Calcutta 700 032, India \\ d3rd Institute of Physics, University of Stuttgart, Pfaffenwaldring 57, 70550 Stuttgart, Germany \\ e-mail: pctc@iacs.res.in
}

\begin{abstract}
Laser-induced fluorescence spectra of a 2:1 complex between 7-azaindole and water, known as 'non-reactive dimer' of the molecule, have been measured in a supersonic jet expansion. The dispersed fluorescence spectrum of the electronic origin band of the complex shows a very large number of low-frequency vibrational features corresponding to different intermolecular modes of the complex in the ground electronic state. Geometries of several possible isomeric structures of the complex and their vibrational frequencies at harmonic approximation were calculated by electronic structure theory method at MP2/6-31G** level. An excellent agreement is observed between the measured and calculated intermolecular vibrational mode frequencies for the energetically most favoured structure of the complex, where the water molecule is inserted within one of the two N...H-N hydrogen bonds of the 7AI dimer.
\end{abstract}

Keywords. LIF spectroscopy; supersonic jet expansion; hydrogen-bonded complex; 7-azaindole; intermolecular vibrational modes.

\section{Introduction}

Hydrogen-bonded complexes of 7-azaindole (7AI) with various protic ligands have attracted much interest in the last few decades for their spectroscopic and photophysical studies. ${ }^{1-35}$ Such species are easily formed in non-polar liquids at room temperature, and upon electronic excitation by an UV light source of wavelength around $300 \mathrm{~nm}$, many of these species emit green fluorescence with band maxima $\left(\lambda_{\max }\right)$ near $500 \mathrm{~nm}$. This unusually large spectral red-shifting between the absorption and emission wavelengths has been ascribed to excited state tautomerization of 7AI, and the green fluorescence is emitted by the tautomeric form. ${ }^{1}$ About two decades ago, it was shown that this excited state process also occurs under a cold supersonic jet expansion environment, and more interestingly the green fluorescence is emitted when the homodimer $\left(7 \mathrm{AI}_{2}\right)$ is excited to $\mathrm{S}_{1}$ zero-point level. ${ }^{19,20}$ It was proposed therefore that the conversion of the locally

\footnotetext{
\#Dedicated to Prof. N Sathyamurthy on his 60th birthday

*For correspondence
}

excited state to the tautomeric configuration occurs by proton/ $\mathrm{H}$-atom tunnelling mechanism, and this was also corroborated by the strong deuterium isotope effect on the tunnelling rate. ${ }^{2,20}$ An apparent reason for the overwhelming interest shown by the spectroscopic community to this system is that the dimer has been considered as a simple prototype for detailed understanding of the light-induced tautomerization processes in the base-pairs of the nucleic acids. ${ }^{36,37}$

The titled water bound complex of 7AI (2:1) shows a very different photophysical behaviour in a supersonic jet expansion environment compared to what is said for the homodimer $\left(7 \mathrm{AI}_{2}\right)$. In the recent past, several research groups have fiercely debated to decide over the actual identity of the complex. Fuke and Kaya were the first to measure its laser-induced fluorescence (LIF) as well as resonance enhanced multi photon ionization (REMPI) spectra. ${ }^{19,20}$ In a LIF excitation spectrum measured under jet-cooled condition, the $S_{1} \leftarrow S_{0}$ transition of the complex appears at a frequency almost similar to that of the pure dimer $\left(7 \mathrm{AI}_{2}\right)$. However, the emission characteristics of the two species are very different, since the pure dimer $\left(7 \mathrm{AI}_{2}\right)$ undergoes facile 
conversion to the tautomeric form in the excited state and emits only visible fluorescence, whereas the complex emits from the locally excited states in ultraviolet region. Consequently, the latter was termed as the 'non-reactive dimer'. ${ }^{19,20}$ To ascertain the identity of the complex, a large number of sophisticated experimental methodologies, like rotationally resolved electronic spectroscopy, ${ }^{24}$ femtosecond coulomb explosion imaging $^{38,39}$ and double resonance IR-induced fluorescence dip spectroscopy ${ }^{28}$ were employed along with electronic structure calculation at different levels of sophistication. An unambiguous mass spectrometric identification of the species suffered due to extensive fragmentation of the 7AI-water complexes upon laserionization. Because of this effect, an electronic or IR spectra recorded by probing a mass species [say 7AI$\left(\mathrm{H}_{2} \mathrm{O}\right)_{\mathrm{n}}$ ] does not guarantee that the recorded spectrum truly correspond to $7 \mathrm{AI}\left(\mathrm{H}_{2} \mathrm{O}\right)_{\mathrm{n}}$. A higher sized cluster, say $7 \mathrm{AI}\left(\mathrm{H}_{2} \mathrm{O}\right)_{\mathrm{m}}$, where $m>n$, can appear at the $7 \mathrm{AI}\left(\mathrm{H}_{2} \mathrm{O}\right)_{n}$ mass channel after fragmentation of the produced cluster ion.

In a number of studies, attempts were made to correlate the 'non-reactive dimer' to a T-shaped homodimeric species $\left(7 \mathrm{AI}_{2}\right)$ where the two moieties are bound mostly via $\pi$-hydrogen bonding interactions. For example, Nakajima et al.,${ }^{24}$ measured the highresolution LIF spectrum of the $\mathrm{S}_{1} \leftarrow \mathrm{S}_{0}$ origin band of the species, and although the rotational structures in the spectrum were not fully resolved, the authors tried to simulate the observed band envelope in terms of a $\mathrm{T}$ shaped dimer geometry. However, electronic structure calculations do not predict any favourable T-shaped isomeric structure for the dimer. T-shaped homo-dimeric species is expected to encounter a large conformational barrier to reorganize to the centro-symmetric form essential for facile tautomerization via double proton/ $\mathrm{H}$-atom exchange between the two dimer moieties.

That the so called 'non-reactive dimer' could contain a bound water molecule was proposed first by Fujii and co-workers by measuring the double resonance fluorescence-dip infrared spectrum. ${ }^{28}$ The spectrum shows a sharp dip at $3708 \mathrm{~cm}^{-1}$, which was correlated with the fundamental of the $\mathrm{O}-\mathrm{H}$ stretching vibration of the free $\mathrm{O}-\mathrm{H}$ group of the water molecule in the complex. A more complicated feature in the IR spectrum near $3000 \mathrm{~cm}^{-1}$ corresponded with the other $\mathrm{O}-\mathrm{H}$ group of the water involved in hydrogen bonding within the complex. However, to our knowledge, since that report no complementary study has been published till date that corroborates with the conclusion of the IR spectroscopic finding.

In the present paper, we report for the first time the vibronically resolved dispersed fluorescence (DF) spec- trum of this so called 'non-reactive dimer' upon excitation of its electronic origin band of the $\mathrm{S}_{1}-\mathrm{S}_{0}$ absorption system. A fluorescence emission spectrum of the complex was reported earlier by Kaya et al., ${ }^{19}$ but because of very poor spectral resolution the authors could not perform any meaningful analysis. By using a sensitive ICCD based detection scheme, we have been successful in measuring resolved fluorescence spectra, which are featured with extremely low-frequency vibrational bands corresponding to various intermolecular modes of the complex. The frequencies of such vibrational modes being manifestation of intermolecular interaction potential, their analysis should provide information about the geometry of the complex. However, composition of the complex being uncertain, we have performed the analysis in the following way. First, assuming that the 'non-reactive dimer' could be an isomeric homodimer, we have fully optimized nine probable isomeric geometries of $7 \mathrm{AI}_{2}$ and vibration frequencies of each of them have been calculated. The same has been done also considering that the species could be a $2: 1$ water complex $\left[(7 \mathrm{AI})_{2}\left(\mathrm{H}_{2} \mathrm{O}\right)\right]$. We show that excellent agreement between experiment and theory occurs if the nonreactive species is a water-bound dimer, i.e., the said 2:1 complex.

\section{Experiment}

The experimental set-up used to record the fluorescence excitation (FE) and DF spectra have been described earlier. ${ }^{40}$ Briefly, the carrier gas helium at a pressure of $2 \mathrm{~atm}$ was passed through heated glass cells containing $7 \mathrm{AI}$ at $80^{\circ} \mathrm{C}$, and the gas mixture was expanded into vacuum through a pulsed nozzle (General Valve, series-9) of orifice with diameter $0.5 \mathrm{~mm}$. The expansion-generated clusters were excited by the frequency-doubled output of a tunable dye laser (Sirah and Plasma Technik, model: Cobra Stretch), which was pumped by the second harmonic $(\lambda=532 \mathrm{~nm})$ of a Nd:YAG laser (Spectra Physics, model: INDI). The laser beam (linewidth $\sim 0.5 \mathrm{~cm}^{-1}$ ) intersects the free jet perpendicularly at about $12 \mathrm{~mm}$ downstream of the nozzle orifice, and the fluorescence was collected from the intersection point in a direction perpendicular to both the laser and the free jet. The FE spectra were measured by detecting the total fluorescence using a Hamamatsu R928 photomultiplier tube. The output signal of the PMT was processed using a boxcar averager (model RS250, Stanford Research Corporation). The averaged output of the boxcar was stored in a computer using a home-built data acquisition system. To measure the dispersed fluorescence spectra we have used a 
$0.75 \mathrm{~m}$ monochromator (Spex, model $750 \mathrm{M}$ ) having a grating of groove density $2400 / \mathrm{mm}$ and a double staged Peltier cooled ICCD (Jovin Yvon, Model NO. 3000 V) detector.

7-azaindole was procured from Aldrich, and purified further by vacuum sublimation before use. For theoretical prediction of various isomeric structures of 7AI homodimer and also of 2:1 water complex $\left[(7 \mathrm{AI})_{2}\left(\mathrm{H}_{2} \mathrm{O}\right)\right]$ in the ground electronic state, geometry optimizations were performed by $a b$ initio theory method at MP2/6-31G** level. Ground state vibrational frequencies of the two said species were also calculated using the same theoretical method. All calculations were performed using G03 program package. ${ }^{41}$

\section{Results and discussion}

Figure 1 shows the three FE spectra for $S_{1} \leftarrow S_{0}$ excitation of the complex. The spectra in the top two panels (panels-a and b) are for detection of UV and visible segments of the fluorescence, and the bottom panel (c) shows the spectrum recorded without the use of any optical filter in front of the PMT detector, i.e., for detection of the total fluorescence. As expected, the band features in the latter spectrum is basically a superposition of those in the two former spectra. The overall appearance of these spectra is almost identical to what was reported earlier by Kaya et al. ${ }^{20}$ The longest wavelength band at $32244 \mathrm{~cm}^{-1}$ in panel-b represents the electronic origin band of the doubly hydrogen bonded centrosymmetric homodimer (see below, figure 3, MSDHB). This band is $\sim 2381 \mathrm{~cm}^{-1}$ redshifted from the $S_{1} \leftarrow S_{0}$ electronic origin band of 7AI monomer. Since it appears prominently in panel-b but completely absent in panel-a, it implies that the tautomeric conversion of 7AI in homodimeric complex occurs exclusively from the $S_{1}$ zero-point level, and the fluorescence is emitted only by the tautomer in the visible region of the spectrum.

In the topmost panel the band labelled $\mathrm{A}_{0}^{0}$ is the origin of the so called 'non-reactive dimer'. The lowfrequency features built-up on the origin band in this spectrum are due to different intermolecular vibrations

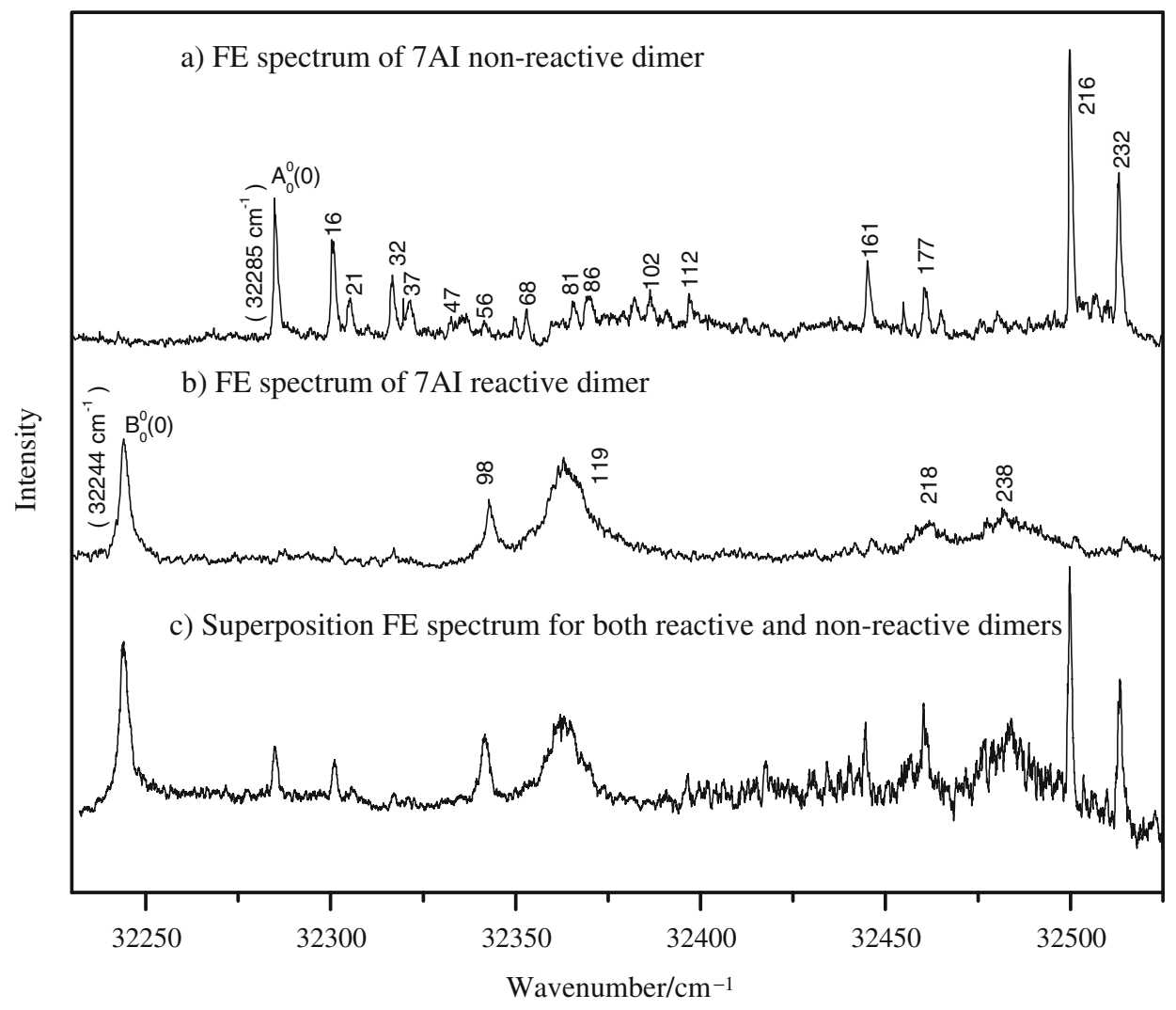

Figure 1. LIF excitation spectra for detection of (a) UV fluorescence, (b) visible fluorescence and (c) total fluorescence of 7AI complexes formed in a supersonic jet expansion. The band labelled $A_{0}^{0}$ is the $S_{1} \leftarrow S_{0}$ electronic origin band of the complex that emits UV fluorescence (non-reactive dimer), and the one labelled $\mathrm{B}_{0}^{0}$ is the electronic origin of the centrosymmetric doubly-hydrogen bonded homodimer of 7AI. In the present study, DF spectrum has been measured by exciting $\mathrm{A}_{0}^{0}$ band. 
of the complex in the excited state. Here a notable point is that although the photophysical behaviour of the two complexes is dramatically different (as said above), their electronic transition energies are nearly same, i.e., the spectral red-shifting of the $\mathrm{S}_{1} \leftarrow \mathrm{S}_{0}$ transition of the 7AI chromophore occurring due to the hydrogen bond formation is almost same. This implies that the nature of hydrogen bonding at the donor-acceptor sites of 7AI in both the complexes must be very similar.

It is worthwhile to point out here the differences in Franck-Condon active vibrational band structures in the top two panels. In the FE spectrum of the reactive dimer (panel-b), the first band appear at $98 \mathrm{~cm}^{-1}$ above the $S_{1}$ origin, and the second band with a maximum at $119 \mathrm{~cm}^{-1}$ appears quite broad. Sekiya and co-workers ${ }^{29}$ have shown earlier that the fluorescence decay time of $119 \mathrm{~cm}^{-1}$ band of the dimer is shorter compared to that of the zero-point level. Therefore, it is likely that the homogeneous factor (shorter lifetime) has a contribution to the broadening effect. The combination band of the two appears at $218 \mathrm{~cm}^{-1}$, and the overtone of the latter appears at $238 \mathrm{~cm}^{-1}$. In an earlier study, the $119 \mathrm{~cm}^{-1}$ band of the homodimer was assigned to the intermolecular stretching fundamental at the hydrogen bonded interface. A direct verification of the assignment cannot be made by use of dispersed fluorescence spectroscopy, because, the emission occurs only from the relaxed excited tautomeric state to the tautomeric ground state. In contrast, the band structure in panel-a of the spectrum shows that the values of the two lowest frequency fundamentals are as small as 16 and $21 \mathrm{~cm}^{-1}$, and both features reappear as combinations on the other prominent $161 \mathrm{~cm}^{-1}$ band. We have discussed below the assignments of these low-frequency bands, which are fundamentals of different intermolecular modes of the complex. We conclude this paragraph with the note that such large differences in intermolecular vibrational frequencies of the two types of complexes are possible only when their compositions are different. It is of course a general fact that large difference in intermolecular vibrational frequency could occur between two isomers if their nature of linkages is different, for example, the case of planar and T-shaped isomers of the dimer. However, in that case, the spectral shift of the $\mathrm{S}_{1} \leftarrow \mathrm{S}_{0}$ transition of 7AI moiety will be very different in the two isomeric complexes, because, the number as well as type of hydrogen bonds in the two cases will be different. But the specific case that we have addressed here is about two species whose spectral shifts are nearly same, i.e., the types of hydrogen bonds at the donor-acceptor sites of 7AI in both complexes are similar as we mentioned earlier.
The dispersed fluorescence spectra (DF) of the nonreactive dimer recorded following excitation of the $\mathrm{A}_{0}^{0}$ band is shown in figure 2. Only the first $500 \mathrm{~cm}^{-1}$ spectral range of the measured spectrum is shown here, and beyond this energy range the signal intensity is extremely low. The overall intensity of the $\mathrm{A}_{0}^{0}$ band being small, for recording of the DF spectrum we have integrated the signals of 9000 laser shots using ICCD. The background produced by stray light and electronic noises has been subtracted by recording blanks, i.e., by keeping the pulsed nozzle off. We also attempted to record DF spectra following excitations of other low-frequency bands of this complex, however, due to poor signal-to-noise ratio no additional information was obtained from those data.

The most interesting feature of the DF spectrum of the complex is appearance of a very large number of extremely low-frequency vibrational bands. It is worth mentioning that in the DF spectrum of the $S_{1}$ origin band of 7AI monomer, the first vibrational band appears at as high as $432 \mathrm{~cm}^{-1}$ corresponding to an in-plane deformation mode of the aromatic ring. ${ }^{19}$ Therefore, we assign the low-frequency features in figure 2 to fundamentals, overtones and combinations of different intermolecular modes of the complex in the ground electronic state. The spectrum has been analysed in the following way. The lowest frequency $16 \mathrm{~cm}^{-1}$ fundamental forms a short but distinct progression, and the first overtone of which appears at $32 \mathrm{~cm}^{-1}$. This

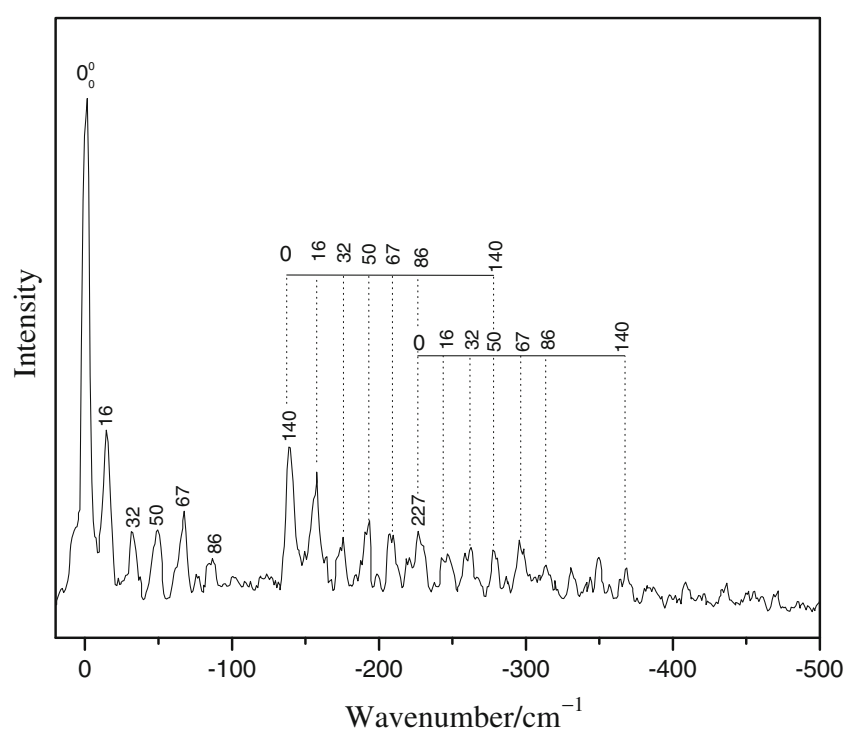

Figure 2. Dispersed fluorescence spectrum recorded upon excitation of the $\mathrm{A}_{0}^{0}$ band of 7-azaindole dimer. This band is known as the origin of the non-reactive dimer of 7AI, which is actually a water bound $7 \mathrm{AI}$ dimer $\left[\left(7 \mathrm{AI}_{2}\right)\left(\mathrm{H}_{2} \mathrm{O}\right)_{1}\right]$. The low-frequency features in the spectrum are due to intermolecular vibrational modes of the complex. 
progression reappears throughout the spectrum as combinations with other fundamentals (shown using dotted lines). It is worth making a comparison of this progression with the one that appears in the FE spectrum (figure 1), where the first band shows up at $16 \mathrm{~cm}^{-1}$ and the size of the progression also appears same. In both spectra, $0_{0}^{0}$ band is most intense, the frequency of the first intermolecular vibrational band is same and the size of the progression for this mode also appears almost same. These similarities indicate that the intermolecular interaction potentials, particularly with respect to this mode in the two electronic states $\left(\mathrm{S}_{0}\right.$ and locally excited state of the complex) are not significantly different. Such similarity in interaction potentials in the two states $\left(S_{1}\right.$ and $\left.S_{0}\right)$ of the complex is consistent with its photophysical behaviour that the species is 'non-reactive' in the excited state. The frequency of the second fundamental in the FE spectrum is $21 \mathrm{~cm}^{-1}$ and it is most likely that the corresponding band in the DF spectrum falls within the profile of the first. The second distinct band in the DF spectrum appears at $50 \mathrm{~cm}^{-1}$ displacement, which can be assigned as the second overtone of the $16 \mathrm{~cm}^{-1}$ fundamental. However, considering its shape, which is relatively broad and has larger intensity, we assign it as superposition of a different vibrational fundamental $\left(\sim 50 \mathrm{~cm}^{-1}\right)$ and the second overtone of the $16 \mathrm{~cm}^{-1}$ band. A fundamental of similar frequency also appears in the FE spectrum. An overlapping band structure is also seen for the $67 \mathrm{~cm}^{-1}$ band in the DF spectrum. The band at $140 \mathrm{~cm}^{-1}$ displacement is the most intense intermolecular fundamental in this spectrum, and the building up of the other low-frequency transitions on the former are shown by dotted lines. The corresponding band in the FE spectrum appears at $161 \mathrm{~cm}^{-1}$. In the FE spectrum, the most intense band appears at $216 \mathrm{~cm}^{-1}$ above the origin, and its counterpart in the DF spectrum appears at $227 \mathrm{~cm}^{-1}$, where the combination tones on it are shown using dotted lines.

As mentioned earlier, a considerable effort has been devoted to explain this 'non-reactive' species as a T-shaped homodimer. We first argue that the observed low-frequency vibrational features in the DF spectrum do not support this assignment. Figure 3

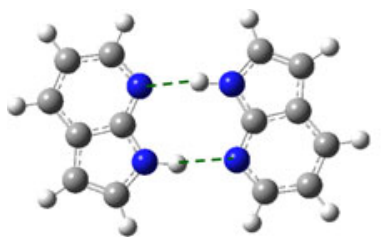

MSDHB (-20.3)

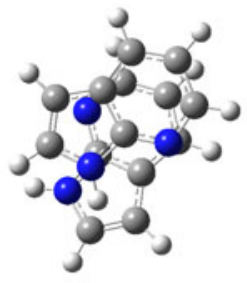

CP3 (-9.2)

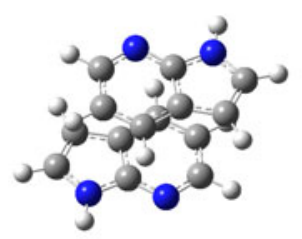

DD (-8.4)

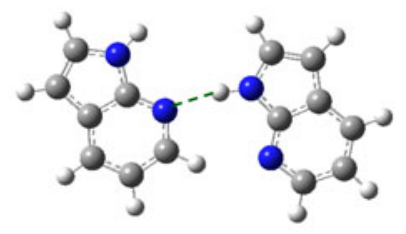

SHBP (-11.6)

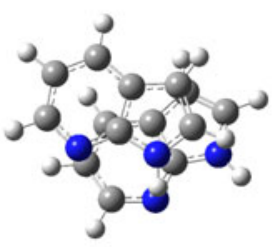

CP4 (-9.0)

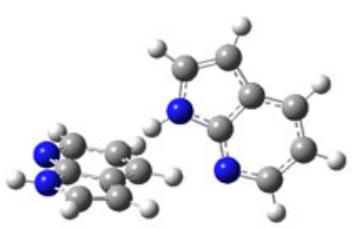

C3T (-8.1)

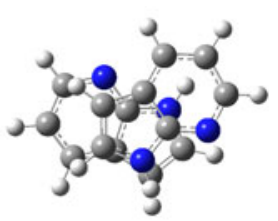

APP (-10.1)

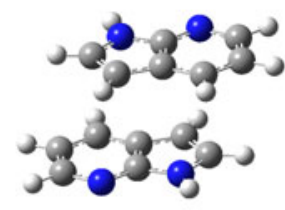

ADD (-8.7)

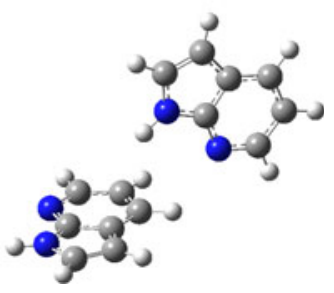

C5T (-6.9)

Figure 3. Nine optimized isomeric structures of 7-azaindole dimer predicted by calculations at MP2/6-31G** level of theory. The explanations of different abbreviations used to label the structures are following. MSDHB = Most stable doubly hydrogen-bonded, SHBP = Single hydrogen-bonded planer, DD = Dipole-Dipole (Ref. 42), CP3 = CardPack 3 (Ref. 42), CP4 = Card-Pack 4 (Ref. 42), ADD = Anti Dipole-Dipole, APP = Anti Pyrrole-Pyrrole, $\mathrm{C} 3 \mathrm{~T}=\mathrm{T}$-shaped at $\mathrm{C}_{3}$ (Ref. 42) and $\mathrm{C} 5 \mathrm{~T}=\mathrm{T}$-shaped at $\mathrm{C}_{5}$. The binding energy $(\mathrm{kcal} / \mathrm{mol})$ of each isomeric species is given in parenthesis. 
Table 1. Predicted fundamental frequencies $\left(\mathrm{cm}^{-1}\right)$ for the intermolecular vibrations of nine isomers of 7-azaindole dimer. Calculations have been performed at $a b$ initio MP2/6-31G** level of theory. The descriptions of the intermolecular vibrational modes of MSDHB and SHBP isomers are presented here.

\begin{tabular}{lcccccccccc}
\hline & & & & & & & & $\begin{array}{c}\text { Measured } \\
\text { intermolecular } \\
\text { fundamentals }\end{array}$ \\
\hline $\begin{array}{l}\text { Intermolecular } \\
\text { vibrations }\end{array}$ & MSDHB & SHBP & DD & CP3 & CP4 & ADD & APP & C3T & C5T \\
$\left(v_{1}\right)$ & 23 [Torsion (OOP)] & 16 [Torsion (OOP)] & 8 & 21 & 18 & 16 & 26 & 15 & 16 & 16 \\
$\left(v_{2}\right)$ & 34 [Butterfly (OOP)] & $25[$ [Butterfly (OOP)] & 42 & 38 & 37 & 39 & 39 & 21 & 19 & - \\
$\left(v_{3}\right)$ & 81 [Opening (IP)] & $45[$ [Opening (IP)] & 50 & 57 & 58 & 62 & 52 & 34 & 24 & 50 \\
$\left(v_{4}\right)$ & $86[$ [Stagger (OOP)] & 69 [Stagger (OOP)] & 76 & 74 & 75 & 75 & 74 & 49 & 47 & 67 \\
$\left(v_{5}\right)$ & 94 [Sliding (IP)] & 71 [Sliding (IP)] & 89 & 92 & 89 & 93 & 86 & 60 & 56 & - \\
$\left(v_{6}\right)$ & 115 [Stretching (IP)] & 104 [Stretching (IP)] & 94 & 111 & 106 & 98 & 108 & 96 & 95 & 140 \\
\hline
\end{tabular}

${ }^{\mathrm{a}} \mathrm{OOP}=$ Out-of-plane

${ }^{\mathrm{b}} \mathrm{IP}=$ In-plane

shows the fully optimized structures of 9 different conformations of the homodimer with their binding energies for calculations performed at MP2/6-31G** level. Some of these structures (SHBP, C3T, MSDHB, DD, CP3 and CP4) were also calculated earlier by Catalan et al., ${ }^{42}$ at DFT/B3LYP/6-31G** and MP2/ 6-31G** level of calculations. To correlate the predicted structures with the observed vibrational features in the DF spectrum, we have calculated the normal mode frequencies of all these isomers using the same theoretical method (MP2/6-31G**). Earlier, we have shown that the method is very effective for accurate prediction of the frequencies of the intermolecular vibrations of diverse 1:1 hydrogen-bonded complexes. ${ }^{43-45}$ Table 1 shows the calculated frequencies for the intermolecular modes of all the nine isomeric dimers. For the two planar structures, all such modes have been defined. However, for other geometries the mode descriptions are not very straightforward, and calculated frequencies are arranged in the table in an ascending order. A comparison of the predicted intermolecular vibrational frequencies with those of the observed fundamentals indicates that there is no comprehensive agreement between theory and experiment with respect to any of the isomeric structures of the homodimer. As an illustration, among the six intermolecular modes the largest frequency is predicted for the stretching vibration between

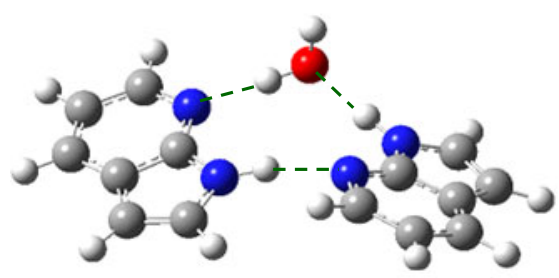

A (0)

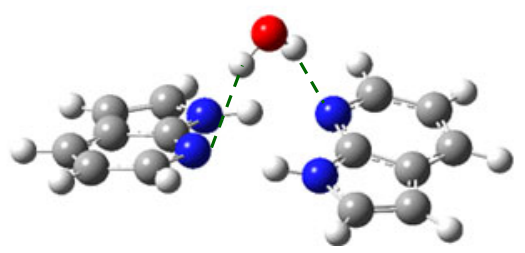

C (4.2)

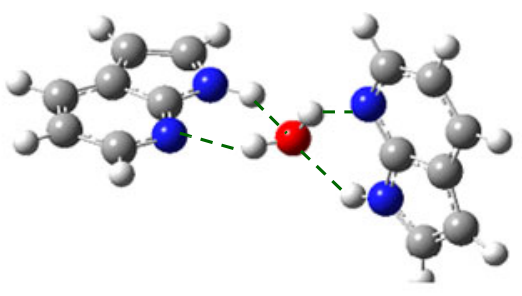

B (2.5)

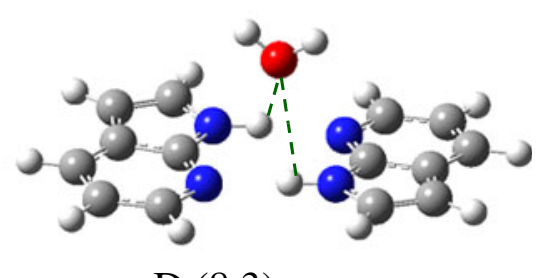

$\mathrm{D}(8.3)$

Figure 4. Four optimized geometries of $(7 \mathrm{AI})_{2}\left(\mathrm{H}_{2} \mathrm{O}\right)_{1}$ [A-D] clusters predicted by calculations at MP2/6-31G** level of theory. The values under the structures present the relative energy difference in $\mathrm{kcal} / \mathrm{mol}$ among the isomers. 
the two dimer moieties, and the values are around $100 \mathrm{~cm}^{-1}$ in all cases. In contrast, the most prominent intermolecular feature in figure 2 appears at $140 \mathrm{~cm}^{-1}$, and the discrepancy with respect to prediction is very large. We would like to mention here that in small hydrogen-bonded molecular complexes, the intermolecular interaction potential is most sensitively reflected in the values of intermolecular stretching mode. Therefore, we suggest that the discrepancy between observation and prediction could be considered as an indication that the species probed by DF spectroscopy is not any isomer of the homodimer $\left(7 \mathrm{AI}_{2}\right)$.

Figure 4 shows the fully optimized structures of four stable isomers of the water-bound complex of 7AI dimer. Their relative stabilities (binding energies) with respect to the most stable isomer (A), where one of the $\mathrm{O}-\mathrm{H}$ bonds of a water molecule is inserted within one of the two $\mathrm{N}-\mathrm{H} \cdots \mathrm{N}$ hydrogen bonds of the 7AI dimer, is also shown. In isomer $\mathrm{B}$, the water molecule is fully inserted between the two 7AI molecules. In this case, each $\mathrm{O}-\mathrm{H}$ bond of water forms a cyclic network with each 7AI molecule making the overall geometry of the complex non-planar. In other two isomeric structures (C and $\mathrm{D}$ ), water is sitting on the top of the doubly hydrogen bonded cyclic interface in two different modes and is also involved in additional hydrogen bonding. Figure 5 shows the intermolecular vibrational modes for the isomer A predicted by calculation at MP2/6-31G** level. It is seen that almost all of the observed intermolecular vibrational fundamentals satisfactorily agree with the predicted frequencies of this complex. We have noted that the frequency calculations at DFT/B3LYP/6-31G** level for isomer (A) also show good agreement with experiment. Thus, the $16 \mathrm{~cm}^{-1}$ band in DF spectrum can be assigned to

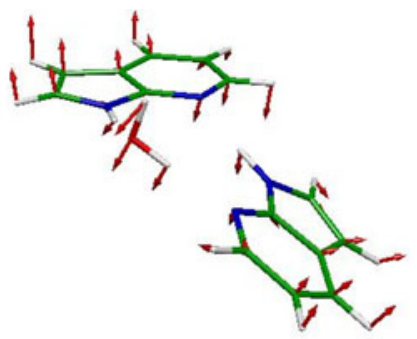

$17 \mathrm{~cm}^{-1}$

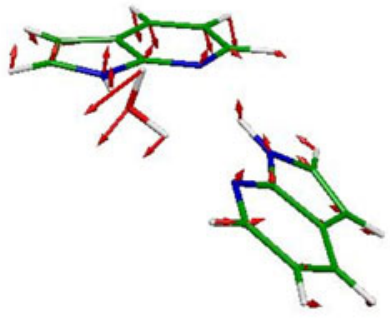

$57 \mathrm{~cm}^{-1}$

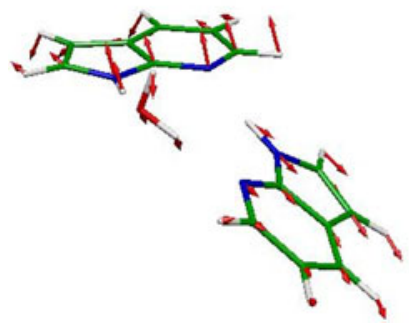

$104 \mathrm{~cm}^{-1}$

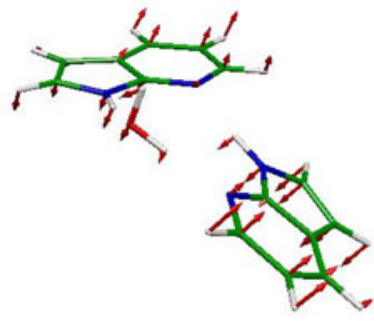

$21 \mathrm{~cm}^{-1}$

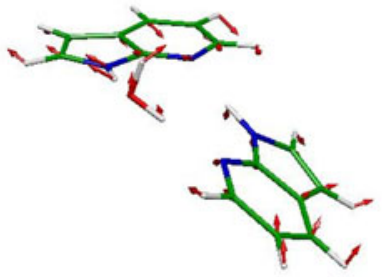

$63 \mathrm{~cm}^{-1}$

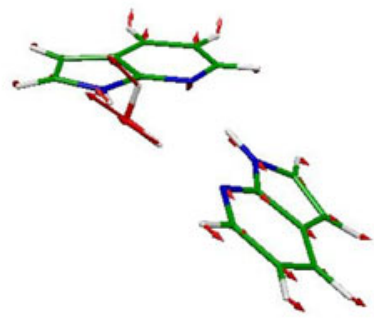

$154 \mathrm{~cm}^{-1}$

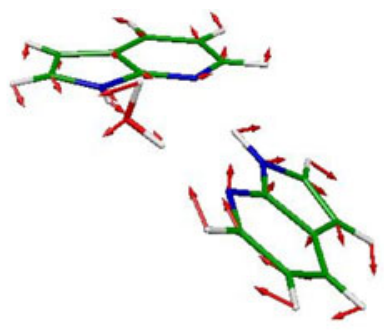

$47 \mathrm{~cm}^{-1}$

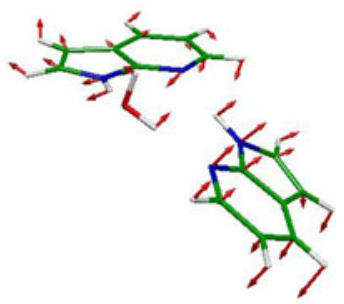

$75 \mathrm{~cm}^{-1}$

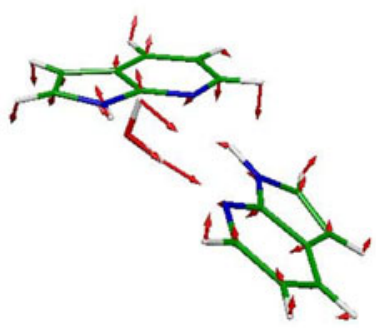

$228 \mathrm{~cm}^{-1}$

Figure 5. Visualization and predicted frequencies of structure A (figure 4) with the composition of $(7 \mathrm{AI})_{2}\left(\mathrm{H}_{2} \mathrm{O}\right)_{1}$ at $\mathrm{MP} 2 / 6-31 \mathrm{G}^{* *}$ level of theory. 
intermolecular out-of-plane butterfly mode. The twisting fundamental, for which the calculated frequency is $21 \mathrm{~cm}^{-1}$, is said earlier to be falling within the band profile of the former, but it appears distinctly in the FE spectrum. The most intense fundamental transition at $140 \mathrm{~cm}^{-1}$ in the DF spectrum can be corresponded confidently with the calculated $154 \mathrm{~cm}^{-1}$ vibration, which is the intermolecular stretching mode at the hydrogen-bonded interface. In many of the earlier studies on the hydrogen-bonded complexes it has been shown that the intermolecular stretching vibration gains the largest intensity in the fluorescence spectra. The observed $228 \mathrm{~cm}^{-1}$ fundamental can be correlated with the calculated $227 \mathrm{~cm}^{-1}$ mode of the complex and the normal mode picture reveals that it involves both outof-plane butterfly-twisting of the 7AI ring as well as gear type motion of a moiety at the hydrogen-bonded interface. On the basis of such comprehensive agreement between theory and experiment we conclude that the probed species is the isomer $\mathrm{A}$ of $(7 \mathrm{AI})_{2}\left(\mathrm{H}_{2} \mathrm{O}\right)$ complex. Furthermore, the frequencies for $\mathrm{O}-\mathrm{H}$ and $\mathrm{N}-\mathrm{H}$ stretching modes predicted in the present study also agree satisfactorily with the IR spectral frequencies of the complex measured by Fujji et al. ${ }^{28}$

\section{Conclusion}

Vibrationally well-resolved DF spectrum of the UV emitting complex of 7AI, which absorb in the same spectral region of the doubly hydrogen-bonded centrosymmetric homodimer in a supersonic jet expansion, has been measured and reported here for the first time. In $\mathrm{S}_{1} \leftarrow \mathrm{S}_{0}$ LIF excitation spectrum, the relative intensities of the vibronic bands of this species appear similar to those of the homodimer which emits visible tautomer fluorescence, indicating that both species are formed in similar ways. Of the two possible suggestions concerning the identity of the UV emitting complex, our analysis of the low-frequency intermolecular vibrations displayed in the measured DF spectrum agree most satisfactorily with a water-bound 7AI dimer $\left[(7 \mathrm{AI})_{2}\left(\mathrm{H}_{2} \mathrm{O}\right)_{1}\right]$ instead of an energetically less favoured isomer of the pure 7AI dimer. Extensive electronic structure calculation was performed to optimize various possible isomeric structures of both the homodimer and the water bound dimer at MP2/6-31G** level. The vibrational frequencies of both species were also calculated by the same method, and those frequencies were extensively used to assign the vibrational bands in the measured DF spectrum. The results presented here indicate that vibrationally resolved DF spectroscopy coupled with electronic structure calculation could be used as a very effective means in deducing the structure of small hydrogen-bonded complexes that can be synthesized in a cold supersonic jet expansion. We also have shown here that for hydrogen-bonded molecular complexes, the intermolecular vibrational frequencies predicted by MP2 or DFT/B3LYP theoretical methods show good agreement with the observed frequencies.

\section{Acknowledgements}

The authors gratefully acknowledge the financial support received from the Department of Science and Technology (DST), Government of India, and partly from the Council of Scientific and Industrial Research (CSIR) to carry out the research. The authors sincerely thank Professor V Eswaran, Department of Mechanical Engineering, IIT Kanpur for generous computational support, and Professor S Manogaran, Department of Chemistry, IIT Kanpur for assistances in electronic structure calculation.

\section{References}

1. Taylor C A, El-Bayoumi M A and Kasha M 1969 Proc. Natl. Acad. Sci. USA 63253

2. Ingham K C and El-Bayoumi M A 1974 J. Am. Chem. Soc. 961674

3. Douhal A, Kim S K and Zewail A H 1995 Nature 378 260

4. Catalán J and Kasha M 2000 J. Phys. Chem. A 104 10812

5. Catalán J 2004 Phys. Chem. Chem. Phys. 64467

6. Takeuchi S and Tahara T 2007 Proc. Natl. Acad. Sci. USA 1045285

7. Sekiya H and Sakota K 2008 J. Photochem. Photobiol. C: Photochem. Rev. 981

8. Collins S T 1983 J. Phys. Chem. 873202

9. Konijnenberg J, Huizer A H and Varma C A G O 1988 J. Chem. Soc. Faraday Trans. 2841163

10. Moog R S and Maroncelli M 1991 J. Phys. Chem. 95 10359

11. Chen Y, Gai F and Petrich J W 1993 J. Am. Chem. Soc. 11510158

12. Chen Y, Gai F and Petrich J W 1994 Chem. Phys. Lett. 222329

13. Kwon O-H, Lee Y-S, Park H J, Kim Y and Jang D-J 2004 Angew. Chem. Int. Ed. 43 5792, and references therein

14. Kwon O-H and Jang D-J 2005 J. Phys. Chem. B 109 8049

15. Chou P-T, Martinez M L, Cooper W C, McMorrow D, Collins S T and Kasha M 1992 J. Phys. Chem. 96 5203, and references therein

16. Chang C-P, Wen-Chi H, Meng-Shin K, Chou P-T and Clements J H 1994 J. Phys. Chem. 988801

17. Chou P-T, Wei C-Y, Chang C-P and Meng-Shin K 1995 J. Phys. Chem. 9911994

18. Suzuki T, Okuyama U and Ichimura T 1997 J. Phys. Chem. A 1017047 
19. Fuke K, Yoshiuchi H and Kaya K 1984 J. Phys. Chem. 885840

20. Fuke K and Kaya K 1989 J. Phys. Chem. 93614

21. Kim S K and Bernstein E R 1990 J. Phys. Chem. 943531

22. Huang Y, Arnold S and Sulkes M 1996 J. Phys. Chem. 1004734

23. Nakajima A, Ono F, Kihara Y, Ogawa A, Matsubara K, Ishikawa K, Baba M and Kaya K 1995 Laser Chem. 15 167

24. Nakajima A, Hirano M, Hasumi R, Kaya K, Watanabe H, Carter C C, Williamson J M and Miller T A 1997 J. Phys. Chem. A 101392

25. Lopez-Martens R, Long P, Solgadi D, Soep B, Syage J and Millie P 1997 Chem. Phys. Lett. 273219

26. Catalán J, del Valle J C and Kasha M 1999 Proc. Natl. Acad. Sci. USA 968338

27. Folmer D E, Wisniewski E S, Stairs J R and Castleman Jr A W 2000 J. Phys. Chem. A 10410545

28. Yokoyama H, Watanabe H, Omi T, Ishiuchi $\mathrm{S}$ and Fujii M 2001 J. Phys. Chem. A 1059366

29. Sakota K, Okabe C, Nishi N, Sekiya H 2005 J. Phys. Chem. A Lett. 1095245

30. Hara A, Sakota K, Nakagaki M and Sekiya H 2005 Chem. Phys. Lett. 40730

31. Brause R, Krugler D, Schmitt M, Kleinermanns K, Nakajima A and Miller T A 2005 J. Chem. Phys. 123 224311
32. Sakota K, Inoue N, Komoto Y and Sekiya H 2007 J. Phys. Chem. A 1114596

33. Sakota K, Kageura Y and Sekiya H 2008 J. Chem. Phys. 129054303

34. Kageura Y, Sakota K and Sekiya H 2009 J. Phys. Chem. A 1136880

35. Koizumi Y, Jouvet C, Norihiro T, Ishiuchi S, DedonderLardeux C and Fujii M 2008 J. Chem. Phys. 129104311

36. Crespo-Hernández C E, Cohen B, Hare P M and Kohler B 2004 Chem. Rev. 1041977

37. Löwdin P D 1966 Adv. Quant. Chem. 2213

38. Folmer D E, Poth L, Wisniewski E S and Castleman Jr A W 1998 Chem. Phys. Lett. 2871

39. Folmer D E, Wisniewski E S, Hurley S M and Castleman Jr A W 1999 Proc. Natl. Acad. Sci. USA 9612980

40. Das A, Mahato K K and Chakraborty T 2001 J. Chem. Phys. 1146107

41. Frisch M J, Trucks G W, Schlegel H B, et al., 2003 GAUSSIAN 03, Revision B.05. Gaussian Inc., Pittsburgh, PA

42. Catalán J, Pérez P, del Valle J C, de Paz J L G and Kasha M 2002 Proc. Natl. Acad. Sci. USA 995793

43. Hazra M K, Samanta A K and Chakraborty T 2006 J. Chem. Phys. 125214302

44. Hazra M K and Chakraborty T 2006 J. Phys. Chem. A 1109130

45. Hazra M K 2009 Chem. Phys. Lett. 47310 